\title{
PROPUESTA DE ALFABETIZACIÓN DE EDUCACIÓN NO FORMAL, PARA HOMBRES, MUJERES Y OBREROS INMIGRANTES NICARAGÜENSES
}

\author{
Lidieth María Núñez Castro ${ }^{l}$ \\ Estudiante egresada de la Maestría en Educación Rural, CIDE-Universidad Nacional \\ Heredia, Costa Rica
}

Recibido: 03 de agosto 2007 • Aprobado: 29 de setiembre 2007

\begin{abstract}
Resumen: El artículo resume los principales hallazgos de la investigación realizada sobre el nivel de alfabetización de mujeres y hombres, obreros inmigrantes nicaragüenses, residentes en Costa Rica; específicamente, madres y padres de familia con niños en la Escuela Gonzalo Monge Bermúdez, del distrito de Pital de San Carlos.

En la investigación se determinan los motivos más relevantes por los que algunos nicaragüenses han inmigrado a Costa Rica. Además, se estipulan algunas necesidades a las que ellos desean dar solución en este país, así como el papel que pueden cumplir los procesos de educación no formal en la vida de estas personas.

Es de clara importancia realizar una propuesta de alfabetización en Educación no formal, que permita a mujeres, hombres y obreros inmigrantes nicaragüenses prepararse y formarse para la vida y el trabajo.

De acuerdo con el proyecto para América Latina y el Caribe, programa de Educación Para Todos, la educación es una de las necesidades básicas: Cada persona -niño, joven o adulto- deberá estar en condiciones de beneficiarse de las oportunidades básicas de aprendizaje. Estas necesidades comprenden tanto herramientas esenciales para el aprendizaje (lectura y escritura, problemas de aprendizaje...), como contenidos básicos, requeridos para que los seres humanos sean capaces de sobrevivir, desarrollen sus capacidades, vivan y trabajen con dignidad, participen plenamente en el desarrollo, mejoren la calidad de sus vidas, tomen decisiones fundamentales y continúen aprendiendo.
\end{abstract}

Palabras clave: Alfabetización, inmigrantes, educación no formal.

Abstract: This article summarizes the main findings of a research on literacy made with immigrant Nicaraguan men and women workers residing in Costa Rica, specifically with parents from students at the Gonzalo Monge School in Pital, San Carlos.

In this investigation, the more relevant motives for these Nicaraguan immigrants to come to Costa Rica are established. In addition, some of their needs living in this country are stipulated as well as the role of informal education in their lives.

\footnotetext{
Licenciada en Enseñaza de la Educación en I y II ciclos, con énfasis en Educación Rural de la Universidad Nacional. Experiencia en Educación primaria en escuelas rurales de Costa Rica, en la zona norte, así como en el trabajo con cooperativas estudiantiles en esa misma zona. Máster en Educación Rural Centroamericana de la Universidad Nacional. Correos electrónicos: lidygsd@hotmail.com / lidygas@ costarricense.co
} 
It is clearly important to design a literacy proposal on informal education that allows immigrant Nicaraguan men and women workers to prepare and educate for life and work.

According to the Project for Latin America and the Caribbean, Education for Everyone program, education is understood as one basic need of the person: every person -child, young or adult- must have the basic opportunity of taking advantage of education. These needs include not only essential tools for learning (such as reading, writing, learning problems...), but also basic learning contents required for human beings to: survive, develop their capacities, live and work with dignity, fully participate on development, improve the quality of their lives, take their fundamental decisions and continue learning.

Key words: Literacy, immigrants, informal education.

La educación no formal que es la modalidad educativa que comprende todas las prácticas y procesos que se desprenden de la participación de las personas en grupos sociales estructurados, deliberadamente educativos, pero cuya estructura institucional no certifica para posciclos escolarizados avalados por el Estado.

Una activa tarea debe llevarse a cabo para modificar las desigualdades educacionales y suprimir las discriminaciones en el acceso a las oportunidades de aprendizaje de los grupos desamparados: Los pobres, los niños de la calle y los niños que trabajan, las poblaciones remotas y rurales, los trabajadores nómadas e itinerantes, los pueblos indígenas, las minorías étnicas, raciales lingüísticas, los refugiados, los desplazados por la guerra y los pueblos invadidos.

El analfabetismo tiene una estrecha correlación con la pobreza; se estima que los 25 países menos desarrollados (donde el ingreso por cabeza es menor de 100 dólares al año) tienen tasas de analfabetismo de más del 80 por ciento. La proporción de mujeres analfabetas está aumentando continuamente, y las tasas de analfabetismo son mayores en las áreas rurales. A pesar del progreso logrado en proveer educación primaria formal y a pesar de la distribución, en algunos casos, de una cuarta parte de su presupuesto nacional a la educación, muchos países en desarrollo se están quedando rezagados en cuanto a proveer la alfabetización para los niños.

\section{GENERALIDADES}

La intensificación de los flujos migratorios de nicaragüenses hacia Costa Rica ha generado, a raíz de la aceleración de los procesos de globalización de la economía mundial, la conformación de un mercado regional de fuerza de trabajo.

Para el año 2000, y según el Instituto Nacional de Encuestas y Censos (INEC, 2001²citado por Cortés, 2006) en Costa Rica, había 226374 inmigrantes nicaragüenses. Una estimación aceptada como muy confiable, por la rigurosidad de la metodología utilizada; es la realizada por Brenes (1999), la cual cifra el número de inmigrantes residentes entre 300 y 340 mil nicaragüenses en 1999.

Las corrientes migratorias de nicaragüenses hacia Costa Rica responden a una "combinación de factores que tienen como telón de fondo una historia de catástrofes naturales, conflictos políticos y desajustes económicos estructurales..." (Organización Internacional para las Migraciones [OIM], 2001, pp. 7-8).

\footnotetext{
El Instituto Nacional de Estadística y Censos -INEC- es el organismo público, de carácter técnico, que unifica la orientación y ejerce la dirección superior de todas las actividades estadísticas oficiales que se realizan en el territorio de la República Argentina. Su creación y funcionamiento está reglamentado por la Ley 17.622 y el Decreto 3110/70, así como el Decreto 1831/93.

La ley le confiere responsabilidad directa en el diseño metodológico, organización y dirección de los operativos nacionales de relevamiento a través de censos y encuestas, la elaboración de indicadores básicos de orden social y económico y la producción de otras estadísticas básicas.
} 
La zona norte del país de Costa Rica alberga una gran mayoría de estas personas, de las cuales, los hombres trabajan en labores agrícolas y las mujeres que se unen a la fuerza laboral se dedican a los quehaceres domésticos en hogares de familias costarricenses. De ellos, el 10,5\% de nicaragüenses no cuenta con educación formal o tiene la primaria incompleta, ha tenido acceso solamente a la educación no formal e informal (OIM, 2001).

El censo de población del 2000 muestra que el 12\% de la población inmigrante nicaragüense no cuenta con ningún nivel de escolaridad formal (INEC, 2001 citado por Cortés, 2006).

Dado que la educación es una herramienta para contar con un mejor nivel de vida, mejores trabajos y mayor remuneración económica, es vital facilitar propuestas de alfabetización para que los adultos y los obreros inmigrantes nicaragüenses, logren alcanzar el nivel de vida que ellos anhelan al emigrar a este país.

Esta aseveración se puede complementar con lo indicado en el (Proyecto Estado de la Región, 2003, p. 66); en el cual se manifiesta que: “...Se calcula que la incidencia [de la pobreza] se reduce en cuatro puntos porcentuales por cada año de estudio, hasta los doce años". Aseveración que confirma, el papel fundamental de la educación en la lucha contra la pobreza, la formación de capital humano y la creación de empleo de calidad". De acuerdo con la información obtenida en la investigación mencionada, entre las principales necesidades de los inmigrantes, la educación ocupa el cuarto lugar en el eslabón de prioridades.

La investigación realizada lleva como finalidad crear una propuesta de alfabetización no formal para mujeres, hombres, y obreros inmigrantes nicaragüenses, ubicados en determinadas zonas del país. Se responde a las propuestas de alfabetización para los grupos desfavorecidos en los acuerdos tomados en la Declaración Mundial sobre Educación Para Todos, (UNESCO/OREAL, 1990, p. 8), y el cual dice, en su artículo 1: "La satisfacción de las necesidades básicas de aprendizaje: Cada persona -niño, joven o adulto- deberá estar en condiciones de beneficiarse de las oportunidades básicas de aprendizaje".

Lo otro, es disponer de un programa en educación no formal que tome en cuenta las particularidades de los participantes, por ejemplo, el horario y tipo de trabajo, las necesidades de éstos y otras más.

Cabe destacar que la educación no formal se ha constituido en parte importante de la actividad de múltiples instituciones y en una práctica que ha tenido que ser asumida como compensatorio de deficiencias del sistema educativo formal y complemento de programas y políticas encaminadas al desarrollo del medio rural.

\section{¿POR QUÉ EMIGRAN LOS NICARAGÜENSES A COSTA RICA?}

De acuerdo con el Estudio Binacional, realizado por la OIM en el año 2001, cinco son las causas que han originado la migración de nicaragüenses a Costa Rica.

- En primera instancia, en 1972, y a causa del terremoto que sufrió Nicaragua, detonó las primeras masas de migraciones nicaragüenses al país costarricense.

- En 1977 hasta 1979, los nicaragüienses inmigraron por la agudización de la guerra civil que enfrentó Nicaragua. 
- En la década de los ochenta, el estallido del gobierno sandinista y las fuerzas de la contra revolución se convierten en el tercer causante de la inmigración nicaragüense, además de la etapa post-conflicto político-militar, entre 1973 y 1997.

- La última causa mencionada es el huracán Mitch, en 1998 (OIM, 2001).

Según Mojica:

En el proceso de democratización, luego de la firma de los Acuerdos de Paz en la región; entre Nicaragua (1990), El Salvador (1992) y Guatemala (1996), fue imposible detener la migración masiva de población nicaragüense a Costa Rica, que tuvo como causas adicionales la aguda crisis política y económica de orden estructural y coyuntural en Nicaragua. A lo anterior, debe agregarse la aplicación de políticas neoliberales como la puesta en marcha de la amplia apertura externa a través del estímulo a las grandes exportaciones. Sin embargo, las políticas de estímulo a la agroexportación no se tradujeron en su dinamización productiva ni en una verdadera modernización económica e institucional... Paradójicamente, esto benefició más a los grandes productores terratenientes que a los pequeños y medianos campesinos, quienes tenían grandes dificultades de acceder a tierras de frontera agrícola.

[Además], se produjo un desplazamiento de casi 10.000 trabajadores del Estado entre 1985 y 1993..., que sumados a la reducción de 77.257 plazas ocupadas por soldados en el Estado para ese mismo año..., saturaron el sector de la economía informal, con la consecuente reducción significativa de la intervención del Estado en las actividades económicas y sociales.

Otro factor que incidió en el proceso migratorio fue la corrupción sistemática de gobiernos: “...todas las consecuencias sociales que conlleva un período de posguerra, condujo al país a la postración económica, al caos político en varias coyunturas y convirtió en insoportables las condiciones de vida de grandes sectores de la población" (Samandú y Pereira, 1996, p. 7, citado por Acuña y Olivares, 2000, p. 9).

El impacto en la población nicaragüense..., [cuya inmigración obedece a] fenómenos naturales como los huracanes Mitch... y Juana..., han revelado la poca capacidad del gobierno de Nicaragua [para] generar estrategias de desarrollo nacional en los planos político, social y económico, capaces de prever y menguar la expulsión de hombres y mujeres de ese país centroamericano a territorio costarricense...

En este... sentido, las condiciones del capitalismo tardío... en la región centroamericana; han sido marcadas por la implementación de Programas de Estabilización y Ajuste Estructural, tratados de libre comercio, entre otras políticas neoliberales. En esta realidad, tales estrategias se han visto beneficiados con el aporte de la mano de obra "barata" de la población nicaragüense que ha llegado a suelo costarricense, como fuerza laboral en busca de mejorar su situación individual y familiar.

Morales y Zepeda (2002) sostienen que las políticas de Ajuste Estructural en Nicaragua han impactado en los niveles de desempleo y en la pérdida del valor adquisitivo del salario, así como en los bajos niveles de inversión y el deterioro en los niveles de prestación de servicios sociales desde el Estado, especialmente en las áreas de salud, educación y vivienda, evidenciando que la mejoría de la economía reflejada en indicadores macroeconómicos no logra convertirse en beneficios para la mayoría 
de la población nicaragüense y tiende a recrear nuevos patrones de apropiación desigual del ingreso.

Otro factor de atracción de la población nicaragüense [lo constituye] las llamadas ventajas comparativas en el plano social, político y económico de las Costa Rica con respecto a Nicaragua. Sin embargo, tales condiciones también han sido catalogadas como "ventajas competitivas de la miseria" de ambos países con respecto al primer mundo, dado que la estrategia de ajuste estructural en Costa Rica se montó sobre la base de la tecnología atrasada [y] grandes desigualdades en la distribución de la propiedad y la riqueza (Mojica, 2003, pp. 4-6).

\section{NECESIDADES EDUCATIVAS}

Las actividades económicas en Costa Rica se han convertido en factores de atracción del proceso migratorio, éstas demandan, por lo general, una fuerza de trabajo poco calificada, o cuyos niveles de calificación, como ocurre con algunas actividades de la construcción y la manufactura, se obtiene en el lugar de trabajo.

En la zona rural los niveles educativos bajos duplican los existentes en la zona urbana, pues si en la primera los emigrantes nicaragüenses con un nivel inferior a primaria completa suman un 62,5\% de la población mayor de 11 años, en la zona urbana este grupo representa un 31,4\%.

Según el censo de población del año 2000, en las zonas rurales el 38,7\% de nicaragüenses tiene la primaria incompleta, y el 20,4\% completa (INEC, 2001). El estudio agrega que la población nicaragüense entre los 18 y los 24 años de edad que asiste a la educación regular, es de un 11,6\%.

La brecha educativa marca una diferencia social relevante entre los nicaragüenses y el resto de la población del país, ya que las cifras de asistencia a la educación son inferiores a los promedios nacionales, de la misma manera que para los hogares de menores ingresos y de menor nivel educativo. Esta comparación se da de igual forma con el promedio de asistencia a la educación con personas de la región Huetar Norte, comparado con la región periférica del país (Castro, 2002).

En el estudio realizado por la CEPAL (1988, pág. 66 citado en Castro, 2002, p. 55) "...la persistencia de la falta de equidades, el acceso de la educación asociada al estrato social de origen, indica que, en gran medida, las oportunidades de bienestar de los actuales jóvenes ya quedaron plasmadas por el patrón de desigualdades prevaleciente en la generación anterior. Esto se traduce en un alto grado de rigidez social, debido a que el escaso nivel de educación alcanzado por muchos jóvenes bloquea su principal canal de movilidad".

Según manifiesta Claudia Rosales (comunicación personal, marzo, 2007) coordinadora de Pastoral Social de la parroquia de Ciudad Quesada, las mujeres y los hombres adultos, los obreros inmigrantes nicaragüenses, necesitan de la educación para conocer sobre sus derechos, sus deberes, para desenvolverse en un país que aunque no es el suyo, les acoge junto con sus familias. Afirma que la educación no es un lujo, no es una alternativa, no es una opción, es una necesidad que no se puede negar, y que por el contrario, se debe ofrecer a aquellas personas que por diversas razones no la obtuvieron en su momento.

El acceso a la educación ha sido una vía privilegiada para acceder a un nivel de bienestar social aceptable. Según diversos estudios de la CEPAL, y tomando como base estimaciones a partir de las Encuestas de Hogares de 12 países latinoamericanos, se considera que a partir de un nivel educativo de 12 años, que corresponde a [primaria] completa, una persona tiene una alta 
probabilidad de ubicarse fuera de la línea de la pobreza mediante el acceso a un empleo productivo y adecuadamente remunerado (CEPAL, 1998, p. 66, citado en Castro, 2002, p. 55).

En el caso de los migrantes nicaragüenses, no sólo existe una diferenciación social relacionada con su condición social de trabajadores manuales con un nivel educativo bajo, sino también una brecha relacionada con la nacionalidad, tan relevante como la primera.

Para ultimar, como afirma Vera (1985): “...la determinación de una necesidad educativa, se traduce en una demanda de condiciones para vivir procesos de aprendizaje en los cuales se modifiquen las formas de pensar, sentir y actuar, como requisito para transformar la relación de los sujetos entre sí y de estos con la naturaleza...” (p. 20).

\section{EDUCACIÓN NO FORMAL}

En "Educación no formal y exclusión en Centroamérica" (Ooijens y Van Kampen, 2000), se rescata, según la UNESCO (2001) que existen muchos tipos de educación no formal distribuidos sobre las áreas productiva, cultural, social y política, donde menciona los siguientes tipos de educación no formal: educación para el trabajo, formación profesional, educación cultural-étnica, educación religiosa...

Dentro de cada tipo de educación no formal, el estudio identifica varias modalidades según grados de formalidad. Así, coloca la oferta de educación no formal en la parte central de un continuo educativo, entre la educación formal por un lado, y la informal por el otro. Además, señala la existencia de una tendencia de gradual formalización en el nivel de muchos tipos de educación no formal identificados.

La educación no formal se ha constituido, en los últimos tiempos, en parte importante de la actividad de múltiples instituciones y en una práctica que ha tenido que ser asumida como compensatorio de deficiencias del sistema educativo formal y complemento de programas y políticas encaminadas al desarrollo del medio rural.

La clientela de estos programas, en su mayoría, es la población económica y políticamente marginadas: jornaleros, campesinos, pequeños propietarios, amas de casa, etcétera.

Los cursos con ubicación cercana al área de capacitación para el trabajo, no tienen lugar significativo en las áreas rurales. Las características marginales de las zonas rurales contribuyen a que la institución de talleres se torne más difícil, ya que se localizan fundamentalmente en las áreas urbanas. Lo anterior lleva a plantear la casi inexistencia de la capacitación para el trabajo en el medio rural. En educación no formal, se apunta que lo básico de las capacitaciones es que estas den respuesta a las necesidades de las comunidades.

El mismo autor, apunta que es imposible combatir la pobreza con una educación pobre y de baja calidad. Se hace necesario profesionalizar el servicio de educación de adultos y fomentar programas orientados específicamente a la formación de éstos.

De acuerdo con el equipo coordinador de la Maestría en Educación Rural Centroamericana, de la Universidad Nacional, la Educación no Formal se define como el uso de estrategias, técnicas, actividades, metodologías y mediaciones participativas. En esta dinámica, el sujeto es el centro, no el material de trabajo; no existe una calificación numérica, sino una evaluación por procesos, relacionada con objetivos claros, cortoplacistas y pertinentes (de acuerdo con los intereses del participante).

Para quien facilita u organiza procesos educativos no formales en comunidades rurales, la experiencia formativa es enorme; especialmente, porque tales dinámicas evidencian los saberes de 
la población rural. Se trata de facilitadores y dialogadores dispuestos al aprendizaje y el trabajo conjunto. De ahí que una intervención metodológica similar debiera ser implementada en los procesos formales que se ejecutan en esas zonas.

La educación no formal se sustenta en las necesidades reales, cotidianas y prioritarias de una comunidad, y surge desde los planteamientos que esta hace. Por lo general, cuando la educación emerge de esta manera, se obtiene mayor participación y compromiso, y es mucho más fácil ver el surgimiento de procesos de identificación y empoderamiento comunitario, bases de la organización y desarrollo comunal.

Las comunidades, ya empoderadas, satisfacen sus necesidades mediante procesos de gestión, cogestión y autogestión, y desarrollan también condiciones que promueven y facilitan el aprendizaje, la creatividad, la reflexión, la implementación de herramientas y la evolución de potencialidades. Asimismo, se propicia la educación integral, la salud individual, el cooperativismo y la creación de líneas comunicativas y organizacionales para el trabajo con diversas asociaciones, instituciones $\mathrm{u}$ organismos.

De esta manera, el trabajo generado es voluntario, y surge de la identificación y la motivación. El liderazgo y la proyección nacen entonces cuando se gesta un proceso educativo no formal que resulta exitoso.

Por otra parte, la educación no formal suele rescatar, en distintas facetas, las habilidades individuales, las oportunidades y las destrezas colectivas que coexisten en una región. Esto, sin duda, fortalece la autoestima de los locales, y contribuye a afianzar la identificación cultural, el aprecio y el respeto hacia el entorno natural, lo autóctono, los saberes o habilidades sociales y los valores culturales endógenos; se evita con ello la inclusión de actividades, actitudes, supuestos o "paquetes educativos" hechos y aplicados en lugares ajenos.

La educación no formal fortalece las relaciones entre las organizaciones comunitarias, la escuela, las madres y padres de familia y las instituciones locales. Al mismo tiempo, se promueven actitudes de apertura y solidaridad como la equidad, la convivencia intercultural, la igualdad de derechos y el respeto.

Se debe destacar que de manera paralela, estos procesos educativos fomentan la inclusión de género, pues, en muchos casos, mujeres y hombres se involucran en pro del trabajo comunal, ejerciendo, lado a lado, las mismas funciones; en otros, la capacitación va dirigida hacia el fortalecimiento de las capacidades y conocimientos de grupos femeninos, la promoción de la pequeña empresa y el fortalecimiento de la autoestima, vitales en sociedades que durante siglos han marginado (limitando su trabajo o crecimiento intelectual, no reconociendo su aporte laboral, agrediendo, otros) a las mujeres por su condición de género.

En esta misma línea, la educación no formal, por su carácter igualitario y por su implementación como respuesta a necesidades locales, ha llegado a los grupos que suelen quedar al margen de los beneficios de la sociedad: las minorías étnicas, los inmigrantes, los más pobres, las personas con alguna discapacidad, las personas de la tercera edad, las niñas, niños y adolescentes, entre otros.

Por ello, muchas de estas capacitaciones han generado el acceso de estos pobladores a la tecnología, a un segundo idioma y a nuevas posibilidades educativas.

Es importante rescatar algunos elementos en cuanto a las propuestas de educación no formal. Para que un proyecto o capacitación no formal prospere, es vital asegurarse de que se orienta hacia el desarrollo de propuestas sostenibles en el tiempo, el espacio y lo económico. De lo contrario, la comunidad lo resiente, y las secuelas suelen ser dramáticas. 


\section{A MANERA DE PROPUESTA}

Han quedado claras las diferentes situaciones que contribuyen a que un número significativo de nicaragüenses hayan inmigrado y sigan inmigrando a Costa Rica. Queda demostrado, además, que las principales necesidades que consideran los nicaragüenses que viven en este país, no contemplan precisamente, la educación.

De una manera contundente, sugiero como medio de alfabetización para instruir la población que interesa, una propuesta de educación no formal que responda a las necesidades de los involucrados, pero que a su vez contribuya a la preparación educativa de la población implicada.

Esta propuesta valora la posibilidad de colaborar en la organización autogestionaria de grupos de inmigrantes, con el fin de que se organicen para promover y formar pequeñas microempresas o cooperativas, las cuales divulguen y rescaten la cultura del pueblo. Por ejemplo, las comidas, el vestido, los bailes, la música, etcétera.

Algunos de los aspectos que se tomarán en cuenta para la propuesta son los siguientes:

- Utilizar en el proceso de la lectoescritura el método natural integral.

- Cuidar que los materiales como afiches, presentaciones y demás, sean lo más interesante posibles, para los participantes.

- Los temas que se desarrollarán dentro del proceso responderán a las principales necesidades de los involucrados.

- Desarrollar el proceso mediante talleres.

- Desarrollar talleres de organización autogestionaria.

- Dar a conocer, mediante los mismos talleres, los diferentes convenios y leyes que los amparan.

- $\quad$ Educar para el trabajo y para la vida.

- Crear conciencia en los educandos sobre la importancia de la educación en sus vidas.

- Temas sobre el rescate de la cultura.

\section{REFERENCIAS}

Brenes, G. (1999). Estimación del volumen y caracerísticas sociodemográficas de los inmigrantes nicaragüenses. Tesis de maestría para la otención del título de Magíster. Sistema de Estudios de Posgrado, Escuela de Estadística, Universidad de Costa Rica. Costa Rica.

Castro, C. (2002). Migración nicaragüense en Costa Rica: población, empleo y necesidades insatisfechas. San José, Costa Rica: Universidad de Costa Rica, Facultad de Ciencias Sociales.

Cortés, A. (2006). Informe sobre el estado de la comunidad nicaragüense en Costa Rica. San José, Costa Rica.

Instituto Nacional de Estadística y Censos (2001). "IX Censo Nacional de Población y V de Vivienda: Resultados generales". Consultado el dia, mes de año de en http://www.inec.go.cr/ INEC_DIS/Publicaciones/archivos\%20SerieCensa1\%20xls/ResulCenso2000.pdf 
UNESCO-OREALC. (1990). Declaración Mundial sobre Educación para todos. Boletín del proyecto principal de educación en América Latina y el Caribe $N^{o} 21$, pp. 8-26. Santiago, Chile: UNESCO-OREALC.

Mojica, F. J. Instituto de Estudios Sociales en población. Heredia, Costa Rica: Universidad Nacional (IDESPO).

Morales, A. y Castro, C. (2002). Redes transfronterizas, sociedad, empleo y migración entre Nicaragua y Costa Rica. Costa Rica: FLACSO.

Organización Internacional para las Migraciones [OIM]. (2001). Estudio Binacional: Situación migratoria entre Costa Rica y Nicaragua. San José, Costa Rica.

Ooijens, J. y van Kampen, P. (2001). Educación no formal y exclusión social en Centroamérica. En L. Lázaro (ed.). Problemas y desafíos para la educación en el siglo XXI en Europa y América Latina (pp. 147-162 ). Valencia: Universidad de Valencia.

Proyecto Estado de la Región. (2003). Segundo informe sobre desarrollo humano en Centroamérica y Panamá del año 2003. San José, Costa Rica: Proyecto Estado de la Nación.

Vera G., R. (1985, Noviembre). Proyecto principal de educación para Améria Latina y el Caribe. En R. Vera (Ed.), Seminario Técnico Regional sobre Formación de Educadores Polivalentes para Comunidades Rurales. Paipa, Colombia: OREALC-UNESCO. 